\title{
Common Mode Response Effects in Differential Measurements
}

\author{
Gemma Hornero, Óscar Casas and Ramon Pallàs Areny
}

Abstract - In differential measurements, before digitized, the signal is conditioned by a differential or fully differential circuit which output depends on both the differential- and commonmode input voltages. Differential signals carry the desired information but common-mode voltages are a nuisance and their contribution to the output signal becomes an interference which is usually evaluated from the ratio between the respective gains for the differential- and common-mode input voltages, Common-Mode Rejection Ratio (CMRR). Usually, only the modulus of the CMRR is specified for IC differential. In this paper we demonstrate that the common-mode response depends on component tolerance and can be band-pass with gain peaking and positive phase shifts inside differential signal passband. Tolerances as low as $\pm 0.01 \%$ cannot prevent phase shifts close to $+90^{\circ}$. The presence of positive phase shifts in the common-mode gain can be suspected whenever the modulus of the CMRR decreases for frequencies below the $-3 \mathrm{~dB}$ bandwidth of the differential gain but cannot be appraised from that modulus alone. Surprisingly, a high CMRR at low frequencies can worsen that effect. Therefore, common-mode effects in differential circuits can be evaluated only from the separate description of the response to differential and commonmode inputs signals.

Index Terms - Differential measurements, differential signal conditioners, common mode response, common mode gain, common mode voltage 


\section{INTRODUCTION}

Differential measurements involve differential sensors, Wheatstone or ac bridges with sensors in one, two or four arms or sensors (and their conditioners) in separate places and connected to a differential amplifier [1], for example for current, high-voltage or biopotential measurement [2]. Differential or pseudo-differential voltages also arise in four-terminal impedance measurements, and in difference measurements at large, for example in thermal standards of power, to minimize common mode effects in tilt measurements and to reduce interference due to ground loops [3].

Differential signal conditioners can process the voltage difference between two nodes even if none of them is connected to signal ground. Therefore, any undesired voltage simultaneously added to both nodes will ideally be cancelled out upon subtracting their respective voltages.

In practice, however, each of the two voltages is processed (amplified, filtered) before subtracting them. The consequence is that unless both voltages undergo identical processing before being subtracted, the final result will depend not only on their difference but also on their average value, termed common-mode voltage, $v_{\mathrm{c}}$. In circuits with differential input and single-ended output, the output voltage contributed by the input common-mode voltage is described by the so-called common-mode gain $G_{\mathrm{c}}$ and the ratio between the differential-mode gain $G_{\mathrm{d}}$ and $G_{\mathrm{c}}$, termed Common-Mode Rejection Ratio (CMRR) [4], [5], is the most common parameter to describe the effect of $v_{\mathrm{c}}$ because the circuit output can be written as

$$
V_{\mathrm{o}}(s)=G_{\mathrm{d}}(s) \cdot V_{\mathrm{d}}(s)+G_{\mathrm{c}}(s) \cdot V_{\mathrm{c}}(s)
$$

and for low-frequency signals we have

$$
v_{\mathrm{o}}=G_{\mathrm{d}}\left(v_{\mathrm{d}}+\frac{G_{\mathrm{c}}}{G_{\mathrm{d}}} v_{\mathrm{c}}\right)=G_{\mathrm{d}}\left(v_{\mathrm{d}}+\frac{v_{\mathrm{c}}}{\text { CMRR }}\right) .
$$

Therefore, a very high CMRR minimizes the output contribution of low-frequency $v_{\mathrm{c}}$ as compared 
to that of $v_{\mathrm{d}}$.

Nevertheless, the assumptions that lead from Eq. (1) to Eq. (2) hold true only if $v_{\mathrm{d}}$ and $v_{\mathrm{c}}$ have the same frequency and $G_{\mathrm{d}}$ and $G_{\mathrm{c}}$ are real numbers, i.e. they do not contribute any phase shift. Obviously, this is not always the case because, on the one hand, $v_{\mathrm{d}}$ is the signal of interest and $v_{\mathrm{c}}$ can be, say, an interference of unknown frequency. On the other hand, $G_{\mathrm{d}}$ is designed to be constant in the frequency band of interest whereas $G_{\mathrm{c}}$ results from component mismatches and depends on circuit topology. Often, the only information available about $G_{\mathrm{c}}$ is that inferred from the CMRR and $G_{\mathrm{d}}$. Since only the modulus but not the phase characteristic of CMRR is specified or measured, it turns out that $G_{\mathrm{c}}$ can be estimated only at very low frequency where CMRR is constant and its phase is presumed to be zero (or $180^{\circ}$ ). But for ac signals, $G_{\mathrm{c}} \times v_{\mathrm{c}}$ could drive the ensuing stage into a nonlinear region, the transient response could be much longer than the one attributable to the differential-mode response, $G_{\mathrm{c}}$ could be unstable in spite of $G_{\mathrm{d}}$ being stable, and a voltage with uncontrolled phase angle could add up to the desired output. None of these undesired effects can be predicted from $G_{\mathrm{d}}$ and the modulus of CMRR.

In fully differential amplifiers, Eq. (2) also holds if $G_{\mathrm{d}}$ is replaced by $G_{\mathrm{DD}}$ (differential-to-differential gain) and $G_{\mathrm{c}}$ by $G_{\mathrm{DC}}$ (common-mode-to-differential gain), so that now $\mathrm{CMRR}=G_{\mathrm{DD}} / G_{\mathrm{DC}}[4]$. Therefore, the same comments above relative to $G_{\mathrm{c}}$ apply to $G_{\mathrm{DC}}$. For example, the possible instability of a very common fully-differential amplifier due to $G_{\mathrm{DC}}$ was reported long ago [6] and the lengthy transient responses due to $G_{\mathrm{DC}}$ have also been studied for some fully differential filters [7].

In this paper we analyze the common-mode gain of differential circuits built by subtracting the outputs of two equal single-ended circuits, and describe their response to common-mode input signals. First, circuit models are obtained for the frequency response to common-mode signals and 
that response is later experimentally assessed. The results should provide a better understanding of the effects of input common-mode signals and lead to stronger design criteria for highperformance differential signal conditioners.

\section{Common Mode Gain of Differential Circuits}

An expeditious way of designing a differential circuit is by subtracting the output voltages of two equal single-ended circuits, as shown in Fig. 1. This is often the case, for example, when the two voltages to be subtracted are obtained at separate places and each voltage is amplified before being subtracted in a central data acquisition unit. If both circuits have a first-order low pass response,

$$
\begin{aligned}
& G_{1}(s)=G_{1} \frac{\omega_{1}}{s+\omega_{1}} \\
& G_{2}(s)=G_{2} \frac{\omega_{2}}{s+\omega_{2}}
\end{aligned}
$$

then the differential output voltage due to a common-mode input voltage $v_{\mathrm{c}}=\left(v_{1}+v_{2}\right) / 2$ is

$$
V_{\mathrm{o}}(s)=V_{\mathrm{oH}}(s)-V_{\mathrm{oL}}(s)=V_{\mathrm{C}}\left[G_{2}(s)-G_{1}(s)\right]
$$

hence the common-mode-to-differential gain is [4]

$$
G_{\mathrm{DC}}(s)=G_{2}(s)-G_{1}(s)=G_{2} \frac{\omega_{2}}{s+\omega_{2}}-G_{1} \frac{\omega_{1}}{s+\omega_{1}}
$$

and $G_{\mathrm{DC}}(0)=G_{2}-G_{1}=\Delta G$.

If we assume that both the dc gain and the corner frequencies of the two single-ended circuits are mismatched because of manufacturing tolerances $t_{G}$ and $t_{\omega}$, respectively, then in a worst case condition we have

$$
\begin{aligned}
& G_{1}=G_{\mathrm{a}}\left(1-t_{G}\right) \\
& G_{2}=G_{\mathrm{a}}\left(1+t_{G}\right)
\end{aligned}
$$


and, initially,

$$
\begin{aligned}
& \omega_{1}=\omega_{\mathrm{a}}\left(1-t_{\omega}\right) \\
& \omega_{2}=\omega_{\mathrm{a}}\left(1+t_{\omega}\right)
\end{aligned}
$$

where $G_{\mathrm{a}}$ is the average dc gain and $\omega_{\mathrm{a}}$ is the average angular corner frequency. Therefore, $G_{1}<$ $G_{\mathrm{a}}<G_{2}$ and $\omega_{1}<\omega_{\mathrm{a}}<\omega_{2}$. If Eq. (6) and Eq. (7) are introduced into Eq. (5), the result is

$$
G_{\mathrm{DC}}(s)=2 G_{\mathrm{a}} \omega_{\mathrm{a}}\left(t_{G}+t_{\omega}\right) \frac{s+\omega_{\mathrm{a}} \frac{t_{G}\left(1-t_{\omega}^{2}\right)}{t_{G}+t_{\omega}}}{\left(s+\omega_{1}\right)\left(s+\omega_{2}\right)}
$$

that can be rewritten as

$$
G_{\mathrm{DC}}(s)=G_{\mathrm{a}} \omega_{\mathrm{a}}\left(\frac{\Delta G}{G_{\mathrm{a}}}+\frac{\Delta \omega}{\omega_{\mathrm{a}}}\right) \frac{s+\omega_{\mathrm{zc}}}{\left(s+\omega_{1}\right)\left(s+\omega_{2}\right)}
$$

where $\Delta G=G_{2}-G_{1}=2 G_{\mathrm{a}} t_{G}, \Delta \omega=\omega_{2}-\omega_{1}=2 \omega_{\mathrm{a}} t_{\omega}$, and

$$
\omega_{\mathrm{zc}}=\omega_{\mathrm{a}} \frac{t_{G}\left(1-t_{\omega}^{2}\right)}{t_{G}+t_{\omega}}=\omega_{\mathrm{a}} \frac{1-t_{\omega}^{2}}{1+t_{\omega} / t_{G}} .
$$

Therefore, $\omega_{\mathrm{zc}}$ will be different from zero whenever $t_{G} \neq 0$ and proportional to the average corner frequency but independent from the dc gain. Since $t_{G}$ and $t_{\omega}$ in Eq. (7) are both positive, then from Eq. (10) it follows $\omega_{\mathrm{zc}}<\omega_{1}, \omega_{2}$ whenever $t_{G}<1$, which is usually the case, and the larger the ratio $t_{\omega} / t_{G}$, the lower will $\omega_{\mathrm{zc}}$ be, i.e., it will fall well inside the differential circuit passband.

According to Eq. (9), the mismatch between two first-order low-pass systems leads to a bandpass response for $G_{\mathrm{DC}}$ that at $\omega_{\mathrm{zc}}$ raises by $3 \mathrm{~dB}$ with respect to $G_{\mathrm{DC}}(0)=\Delta G$, stops raising below $\omega_{1}$, and starts decreasing below $\omega_{2}$. Therefore, $G_{\mathrm{DC}}(\mathrm{s})$ will have a maximum between $\omega_{1}$ and $\omega_{2}$ that will increase with increasing $\Delta \omega$, hence $t_{\omega}$. By equating to zero the first derivative of the modulus of Eq. (9), it is found that the maximum occurs at 


$$
\omega_{\max }=\sqrt{\sqrt{\omega_{\mathrm{zc}}^{4}-\omega_{\mathrm{zc}}^{2}\left(\omega_{1}^{2}+\omega_{2}^{2}\right)+\omega_{1}^{2} \omega_{2}^{2}}-\omega_{\mathrm{zc}}^{2}}
$$

and, for small $\omega_{\mathrm{zc}}$, we have $\omega_{\max } \approx\left(\omega_{1} \omega_{2}\right)^{1 / 2}=\omega_{\mathrm{g}}$, the geometric mean of $\omega_{1}$ and $\omega_{2}$. The maximal gain relative to the dc gain is

$$
\frac{\left|G_{\mathrm{DC}}\right|_{\max }}{\left|G_{\mathrm{DC}}(0)\right|}=\left(1+\frac{t_{\omega}}{t_{G}}\right) \frac{\omega_{\mathrm{a}} \sqrt{\omega_{\max }^{2}+\omega_{\mathrm{zc}}^{2}}}{\sqrt{\omega_{\max }^{2}+\omega_{1}^{2}} \sqrt{\omega_{\max }^{2}+\omega_{2}^{2}}}
$$

which increases with $t_{\omega} / t_{G}$ hence for smaller $\omega_{\mathrm{zc}}$. If $\omega_{\mathrm{zc}}$ is small enough, then the maximal gain is

$$
\left|G_{\mathrm{DC}}\right|_{\max } \approx G_{\mathrm{a}}\left(t_{G}+t_{\omega}\right)
$$

meaning that dc gain and corner frequency tolerance effects add each other. The phase characteristic of $G_{\mathrm{DC}}$ will be positive from below $\omega_{\mathrm{zc}}$ up to some frequency between $\omega_{1}$ and $\omega_{2}$ hence within the passband of the differential gain.

For $\omega_{\max }$ to be real, the radicand in Eq. (11) must be positive, which leads to the conditions

$$
\omega_{1}^{2} \omega_{2}^{2}>\omega_{\mathrm{zc}}^{2}\left(\omega_{1}^{2}+\omega_{2}^{2}\right)
$$

and

$$
\left(t_{G}+t_{\omega}\right)^{2}>2 t_{G}^{2}\left(1+t_{\omega}^{2}\right)
$$

Solving for $t_{\omega}$ yields

$$
t_{\omega}>\frac{t_{G}}{1-2 t_{G}^{2}}\left(\sqrt{2} \sqrt{1-t_{G}^{2}}-1\right)
$$

that for $t_{G}<<1$ can be simplified into $t_{\omega}>(\sqrt{ } 2-1) t_{G} \approx 0.4 t_{G}$, meaning that $G_{\mathrm{DC}}$ peaking is determined by corner frequency mismatch relative to dc gain mismatch.

For matched dc gains $\left(t_{G}=0\right), G_{1}=G_{2}=G_{\mathrm{a}}$, we have $\omega_{\mathrm{zc}}=0$ and Eq. (9) reduces to

$$
\left.G_{\mathrm{DC}}(s)\right|_{t_{G}=0}=G_{\mathrm{a}} \frac{\left(\omega_{2}-\omega_{1}\right) s}{\left(s+\omega_{1}\right)\left(s+\omega_{2}\right)}
$$


which implies a positive phase response from dc up to frequencies above $\omega_{1}$, which is a wider range than that when $t_{G} \neq 0$. The maximal gain occurs now at $\omega_{\mathrm{g}}$ and its value is $G_{\mathrm{a}} t \omega$, obviously smaller than that when $t_{G} \neq 0$ Eq. (13).

If the two corner frequencies are matched instead, $\omega_{1}=\omega_{2}\left(t_{\omega}=0\right)$, then we have $\omega_{\mathrm{zc}}=\omega_{\mathrm{a}}=\omega_{1}=$ $\omega_{2}, \omega_{\max }=0$ and Eq. (9) simplifies to

$$
\left.G_{\mathrm{DC}}(s)\right|_{t_{\omega}=0}=\left(G_{2}-G_{1}\right) \frac{\omega_{\mathrm{a}}}{s+\omega_{\mathrm{a}}}
$$

which is a low-pass response hence has negative phase shift. Therefore, corner frequency mismatch yields the worst $G_{\mathrm{DC}}$ because it makes $G_{\mathrm{DC}}$ to increase inside the differential gain passband and can contribute positive phase shifts in a system which differential-mode response is low pass hence has negative phase shift for differential signals. This can be especially dangerous in ac measurements where in-phase and quadrature components must be separately processed, for example in complex impedance measurements.

If gain tolerances are those in Eq. (6), i.e. $G_{1}<G_{\mathrm{a}}<G_{2}$, but the tolerances of the angular corner frequency are such that

$$
\begin{aligned}
& \omega_{1}=\omega_{\mathrm{a}}\left(1+t_{\omega}\right) \\
& \omega_{2}=\omega_{\mathrm{a}}\left(1-t_{\omega}\right)
\end{aligned}
$$

i.e. $\omega_{1}>\omega_{\mathrm{a}}>\omega_{2}$, then Eq. (9) should be replaced by

$$
G_{\mathrm{DC}}(s)=G_{\mathrm{a}} \omega_{\mathrm{a}}\left(\frac{\Delta G}{G_{\mathrm{a}}}-\frac{\Delta \omega}{\omega_{\mathrm{a}}}\right) \frac{s+\omega_{\mathrm{zc}}^{\prime}}{\left(s+\omega_{1}\right)\left(s+\omega_{2}\right)}
$$

where

$$
\omega_{\mathrm{zc}}^{\prime}=\omega_{\mathrm{a}} \frac{t_{G}\left(1-t_{\omega}^{2}\right)}{t_{G}-t_{\omega}}=\omega_{\mathrm{a}} \frac{1-t_{\omega}^{2}}{1-t_{\omega} / t_{G}} .
$$


Now, whenever $t_{G}<t_{\omega}, G_{\mathrm{DC}}(s)$ will be negative and have a positive zero in the complex plane, which added to the two negative poles in the denominator amount to a maximum $270^{\circ}$ phase shift.

For the particular cases with matched dc gains $\left(t_{G}=0\right)$ or corner frequencies $\left(t_{\omega}=0\right)$, the results are the same obtained before, i.e. Eq. (17) and Eq. (18) respectively.

If $t_{G}=t_{\omega}=t$, which implies matched gain-bandwidth products, $G_{1} \omega_{1}=G_{2} \omega_{2}$, then, from Eq. (5), the result is

$$
\left.G_{\mathrm{DC}}(s)\right|_{t_{G}=t_{\omega}=t}=G_{\mathrm{a}} \omega_{\mathrm{a}}\left(1-t^{2}\right) \frac{\omega_{2}-\omega_{1}}{\left(s+\omega_{1}\right)\left(s+\omega_{2}\right)}
$$

which is an overdamped second-order low-pass response hence shows negative phase shift and no gain peaking.

The differential gain is also affected by gain and corner frequency mismatches as we have [4]

$$
G_{\mathrm{DD}}(s)=\frac{G_{1}(s)+G_{2}(s)}{2}=\frac{1}{2}\left(\frac{G_{1} \omega_{1}}{s+\omega_{1}}+\frac{G_{2} \omega_{2}}{s+\omega_{2}}\right)
$$

and considering tolerances as in Eq. (6) and Eq. (7), it results

$$
G_{\mathrm{DD}}(s)=G_{\mathrm{a}} \omega_{\mathrm{a}}\left(1+t_{G} t_{\omega}\right) \frac{s+\omega_{z \mathrm{~d}}}{\left(s+\omega_{1}\right)\left(s+\omega_{2}\right)}
$$

wherein

$$
\omega_{\mathrm{zd}}=\omega_{\mathrm{a}} \frac{1-t_{\omega}^{2}}{1+t_{G} t_{\omega}}
$$

which, for $t_{G}, t_{\omega}<1$, it implies $\omega_{1}<\omega_{\mathrm{zd}}<\omega_{\mathrm{a}}<\omega_{2}$, and $\omega_{\mathrm{zd}}>\omega_{\mathrm{zc}}$. Therefore, the effect of mismatches is here a reduced gain and phase shift between $\omega_{1}$ and $\omega_{2}$ but otherwise the expected first-order low-pass response is obtained. Dividing Eq. (24) by Eq. (9) yields

$$
\mathrm{CMRR}=\frac{G_{\mathrm{DD}}(s)}{G_{\mathrm{DC}}(s)}=\frac{1+t_{G} t_{\omega}}{2\left(1+t_{\omega} / t_{G}\right)} \frac{s+\omega_{\mathrm{zd}}}{s+\omega_{\mathrm{zc}}}
$$

and $\operatorname{CMRR}(0)=G_{\mathrm{a}} / \Delta G$, which is quite plausible. Since, from Eq. (25) and Eq. (10) (or Eq. (21)), 
$\omega_{\mathrm{zd}}>\omega_{\mathrm{zc}}, \omega_{\mathrm{zc}}^{\prime}, \mathrm{CMRR}$ will decrease by $3 \mathrm{~dB}$ with respect to $\mathrm{CMRR}(0)$ at $\omega_{\mathrm{zc}}$ and will stay constant up to a frequency a bit higher than $\omega_{z d}$ provided the first-order model for each channel in Fig. 1 is still valid at those frequencies. Coincidentally, this is the specified variation of the CMRR with frequency for the INA121 instrumentation amplifier (later used in this work). Since all data sheets of IC instrumentation amplifiers show that CMRR decreases starting at frequencies well below the corner frequency for the differential gain, this means that the common-mode gain starts to increase at low frequencies, the same as for the differential circuit in Fig. 1 in cases Eq. (9), Eq. (17) and Eq. (20), that is, whenever the corner frequencies or the gain-bandwidth products are mismatched, because when $t_{\omega}=0$ or $t_{G}=t_{\omega}$ the common mode response is low-pass (Eq. (18) or Eq. (22), respectively).

Table I summarizes the above scenarios for $G_{\mathrm{DC}}$. Subtracting the outputs from two mismatched first-order low-pass circuits yields a common-mode-to-differential gain with band-pass or lowpass frequency response when the two corner frequencies are mismatched, and a first-order lowpass response if only dc gains are mismatched. If the corner frequency mismatch is larger than about $40 \%$ of the gain mismatch, then the band-pass response Eq. (9) has gain peaking and positive phase angle inside the differential passband, starting at relatively low frequency. If the corner frequency mismatch is smaller than about $40 \%$ of the mismatch between dc gains, then $G_{\mathrm{DC}}$ is first-order, low-pass. If gain-bandwidth products are matched, then $G_{\mathrm{DC}}$ is second-order low-pass Eq. (22). Since the conditions $t_{\omega}=0$ and $t_{G}=t_{\omega}$ are quite improbable, and de gain tuning yields $t_{G}$ $=0$, we will have $t_{\omega}>t_{G}$, so that we can expect a band-pass response for $G_{\mathrm{DC}}$ to be quite common.

\section{EXPERIMENTAL DESIGN}

The mathematical model above has been assessed by implementing the circuit in Fig. 1 with two single-ended amplifiers which outputs are subtracted by measuring their difference with an 
accurate instrumentation amplifier (INA121P), differential gain $G=6$ (Fig. 2), connected to an 8bit Digital Storage Oscilloscope (TDS5054). Phase shift was measured from the time interval between sine wave peaks on the oscilloscope. The circuit was supplied at $\pm 15 \mathrm{~V}$. First, the common mode gain $G_{\mathrm{c}}$ for the INA121P instrumentation amplifier was measured from $1 \mathrm{~Hz}$ to $10 \mathrm{kHz}$ by applying a $10 \mathrm{~V}$ sine wave input in common mode. Below $80 \mathrm{~Hz}$, the output signal was too small to reliably measure the phase shift. The module of $G_{\mathrm{c}}$ increased from $1.4 \times 10^{-4}$ at $1 \mathrm{~Hz}(\mathrm{CMRR} \approx$ $92 \mathrm{~dB})$ to $1.6 \times 10^{-4}$ at $10 \mathrm{~Hz}(\mathrm{CMRR} \approx 91 \mathrm{~dB})$ and $5.4 \times 10^{-3}$ at $10 \mathrm{kHz}(\mathrm{CMRR} \approx 60 \mathrm{~dB})$. These values fall between the specified CMRR for the INA121P for $G=10$, (minimum $91 \mathrm{~dB}$, typical $100 \mathrm{~dB}$ at low frequencies, and $75 \mathrm{~dB}$ typical at $10 \mathrm{kHz}$ ) and $G=1$ (between $78 \mathrm{~dB}$ to $86 \mathrm{~dB}$ at low frequencies and $50 \mathrm{~dB}$ typical at $10 \mathrm{kHz}$ ). The measured phase shift for $G_{\mathrm{c}}$ was $90^{\circ}$ for frequencies below $3 \mathrm{kHz}$, decreasing to $85^{\circ}$ at $10 \mathrm{kHz}$, and $0^{\circ}$ at $320 \mathrm{kHz}$. With these $G_{\mathrm{c}}$ values, and a $1 \mathrm{~V}$ common mode input voltage, the instrumentation amplifier contribution to the output signal is from $150 \mu \mathrm{V}$ for frequencies below $100 \mathrm{~Hz}$ to $5.4 \mathrm{mV}$ maximum at $10 \mathrm{kHz}$. Note that this behavior for $G_{\mathrm{c}}$ is the same obtained above for the case $t_{G} \neq 0, t_{\omega}>0.4 t_{G}$, Eq. (26), in spite that the INA121P does not certainly comprise two separate single-ended stages which outputs are subtracted.

Next, several resistors and capacitors were measured and specific units were selected to implement four different scenarios that could arise in practice because of component tolerances. Table II shows those four cases. Case 1 and Case 2 differ only in $C$ value. Case 3 and Case 4 involve the same components but the positions of $C$ and $C^{\prime}$ are interchanged.

The circuit parameters corresponding to the component values in Table II are shown in Table III. Cases 1 and 2 have different $t_{\omega} / t_{G}$ (less than 0.4 in Case 1, larger than 0.4 in Case 2), but otherwise $G_{2}>G_{1}$ and $\omega_{2}>\omega_{1}$ in both cases. Cases 3 and 4 have $G_{2}>G_{1}$ and similar $t_{\omega} / t_{G}$ but $\omega_{2}$ 
$>\omega_{1}$ in Case 3 and $\omega_{2}<\omega_{1}$ in Case 4.

\section{EXPERIMENTAL RESULTS AND DISCUSSION}

Fig. 3 shows the modulus (top) and phase (bottom) of $G_{\mathrm{DC}}$ in Cases 1 and 2. Theoretical values correspond to the solid and broken lines and experimental results, after discounting the gain of the instrumentation amplifier in Fig. 2, are triangles (Case 1) or squares (Case 2). At low frequencies, $G_{\mathrm{DC}}$ should be close to $\Delta G=0.4$ in both cases and the asymptotes in Fig. 3 are $-8 \mathrm{~dB}(=0.4)$ for the modulus, and $0^{\circ}$ for the phase, as expected. Case 1 leads to a low-pass response because $t_{\omega} / t_{G}$ $=0.055<0.4\left(\right.$ Table I). Case 2, however, with $t_{\omega} / t_{G}=2.5>0.4$ should result in a band-pass response with gain peaking and positive phase shift. The modulus in Fig. 3 shows obvious gain peaking, which cannot belong to an underdamped second-order low-pass system because the transfer function has two distinct negative poles. The phase characteristic shows positive values hence actually corresponds to a band-pass system Eq. (9). The maximal gain is at about $100 \mathrm{~Hz}$, the corner frequency of the differential response, and the ratio $G_{\mathrm{DC}(\max )} / G_{\mathrm{DC}(0)}$ is $1.75(4.87 \mathrm{~dB})$, i.e. very close to the theoretical values: $96 \mathrm{~Hz}$ (from Eq. (11)) and 1.82 (5.23 dB) (from Eq. (12)), respectively. The zero $\left(f_{\mathrm{zc}}\right)$ in the numerator of $G_{\mathrm{DC}}$ is close to one third of $f_{\mathrm{a}}$, as expected. At $10 \mathrm{kHz}, G_{\mathrm{DC}}$ is less than $-40 \mathrm{~dB}$. If the input voltage is $1 \mathrm{~V}$, the output voltage of the instrumentation amplifier is about $60 \mathrm{mV}$, so the $5.4 \mathrm{mV}$ due to the common mode voltage of the INA121P instrumentation amplifier is less than $10 \%$ of the $60 \mathrm{mV}$. For frequencies below $1 \mathrm{kHz}$ this deviation is less than $0.1 \%$ in all cases studied.

From Eq. (10), to obtain a $G_{\mathrm{DC}}$ with band-pass response and a very noticeable gain peak it should be $t_{\omega} / t_{G} \gg 1$ and this can result from a large $t_{\omega}$, a small $t_{G}$, or both. Cases 3 and 4 have a small $t_{G}$ $(0.3 \%)$ and a large $t_{\omega}$ (about $\left.19 \%\right)$ hence fulfill that condition. $G_{\mathrm{DC}}$ in Case $3\left(G_{2}>G_{1}, \omega_{2}>\omega_{1}\right)$ is given by Eq. (9) whereas $G_{\mathrm{DC}}$ in Case $4\left(G_{2}>G_{1}, \omega_{2}<\omega_{1}\right)$ is given by Eq. (20). Since 
$t_{\omega} / t_{G}>>1$, the modulus of Eq. (9) and (20) should be very close and $G_{\mathrm{DC}}(0)=0.003(-50 \mathrm{~dB})$, but the phase characteristic should be very different because of the different sign for $\omega_{\mathrm{zc}}$ and $\omega_{\mathrm{zc}}$ in Eq. (10) and Eq. (21), respectively. Fig. 4 (left) shows that both modulus are about the same and $G_{\mathrm{DC}}(1 \mathrm{~Hz})=-22.5 \mathrm{~dB}$ and decreases with frequency, which is compatible with the expected $G_{\mathrm{DC}}(0)$ $\approx-50 \mathrm{~dB}$. Moreover, Eq. (11) and Eq. (12) predict $\omega_{\max }=570 \mathrm{rad} / \mathrm{s}\left(f_{\max }=91 \mathrm{~Hz}\right)$ and $G_{\mathrm{DC}(\max )} / G_{\mathrm{DC}(0)}=32.20(=30 \mathrm{~dB})$, respectively, and in Fig. 4 we have $f_{\max }=90 \mathrm{~Hz}$, and $G_{\mathrm{DC}(\max )} / G_{\mathrm{DC}(1 \mathrm{~Hz})} \approx 17(\approx 25 \mathrm{~dB})$, which are in good agreement with those predictions. Further, $f_{\mathrm{zc}}$ is much lower than in Case 2. Therefore, whereas $t_{\omega} / t_{G}>1$ yields a large gain peaking, if $t_{G}$ is small then $G_{\mathrm{DC}}(0)$ is so small that $G_{\mathrm{DC}(\max )}$ is a bit larger than 1 and, since $G_{\mathrm{a}} \approx 10$, the result is a poor $\mathrm{CMRR}<20 \mathrm{~dB}$. Therefore, tuning the dc gain of each channel in order to match $G_{1}=G_{2}$ will reduce $G_{\mathrm{DC}}(0)$, which is advantageous at low frequencies, but at the cost a gain peak close to $G_{\mathrm{a}} t_{\omega}$ and, according to Eq. (17), large positive phase shifts at frequencies well below the corner frequency of the differential mode response. Similar behaviors of the common mode response to those presented in Fig. 4 had been previously observed experimentally in differential circuits [13]. It was known that the cause was the tolerance of the design resistances, but they had not been modeled and quantified theoretically.

The agreement between theoretical predictions and experimental results for $G_{\mathrm{DC}}$ is good enough for the equations above to be used to estimate $G_{\mathrm{DC}}$, and $\mathrm{CMRR}$, for usual tolerance values for gain and corner frequency in order to assess their impact. Table IV shows three different cases, all with $0.01 \% \mathrm{dc}$ gain tolerance, which is very good. In case $\mathrm{A}, t_{\omega}=10 \%$, which can easily result, for example, from capacitor tolerance in passive filters, yields a zero in $G_{\mathrm{DC}}$ near $1 \mathrm{~Hz}$ that makes it to increase from $-54 \mathrm{~dB}$ to $0 \mathrm{~dB}$ at $21 \mathrm{~Hz}$, with a phase shift of $85^{\circ}$ at this frequency. As a result, CMRR decreases from $74 \mathrm{~dB}$ at dc to $17 \mathrm{~dB}$ at $1 \mathrm{kHz}$, and maximal phase shift of $-86^{\circ}$ at $30 \mathrm{~Hz}$. 
Therefore, CMRR can be considered a real number only well below $1 \mathrm{~Hz}$ ! Increasing the dc gain by $10\left(G_{\mathrm{a}}=100\right.$, case B), worsens $G_{\mathrm{DC}}(0)$ from $-54 \mathrm{~dB}$ to $-34 \mathrm{~dB}$, and consequently $G_{\mathrm{DC} \text {,max }}$ from $0 \mathrm{~dB}$ to $20 \mathrm{~dB}$, but the other parameters remain the same. This worsening of $G_{\mathrm{DC}}$ is a direct consequence of the use of two separated stages which only interconnection is through signal ground. If the dc gain is kept at 100 but $t_{\omega}$ is improved to $1 \%$, then $t_{\omega} / t_{G}$ becomes 10 times smaller and the effect is an upwards shift of the zero of $G_{\mathrm{DC}}$ to $9.9 \mathrm{~Hz}$ and, whereas $G_{\mathrm{DC}}(0)$ remains at $34 \mathrm{~dB}, G_{\mathrm{DC} \text {,max }}$ is now only $0 \mathrm{~dB}$ and CMRR at $1 \mathrm{kHz}$ improves by $20 \mathrm{~dB}$. Nevertheless, the maximal phase shift of $G_{\mathrm{DC}}$ is $74^{\circ}$, hence considerable, and the maximal phase shift of CMRR is $79^{\circ}$ at $96 \mathrm{~Hz}$, still well below $1 \mathrm{kHz}$.

A further reduction of $t_{\omega}$ while $G_{\mathrm{a}}$ is kept at 10 , as in case A, should improve everything with respect to case A, exception made of $G_{\mathrm{DC}}(0)$ and $\mathrm{CMRR}(0)$. Case D in Table IV shows that improving $t_{\omega}$ by 100 increases $f_{\mathrm{zc}}$ by about $90, G_{\mathrm{DC}(\max )}$ and $\mathrm{CMRR}$ at $1 \mathrm{kHz}$ by about $40 \mathrm{~dB}$, and $f\left(\phi_{\mathrm{DC}(\max )}\right)$ by $170 \mathrm{~Hz}$, which are all beneficial, and decreases $\phi_{\mathrm{DC}(\max )}$ by $43^{\circ}$, which is also good. Nevertheless, CMRR $(0)$ is still poor for the excellent tolerances $\left(0.01^{\circ} \%\right.$ for the gain and $0.1^{\circ \%}$ for the corner frequency, and the $43^{\circ}$ phase shift for $G_{\mathrm{DC}}$ at $191 \mathrm{~Hz}$, hence well below the circuit passband $(1 \mathrm{kHz})$ can contribute major errors in the imaginary part of complex quantities if the common-mode voltage is relatively large as compared to the differential-mode voltage.

It could be argued that since the circuit in Fig. 2 comprises independent stages for each input signal, and it is well known that this yields a very poor CMRR [8], high-performance differential signal conditioners, and integrated circuits in particular, should rely on better topologies that avoid common-mode gain peaking and positive phase shifts. However, that topology is very common in differential measurements with distant sensors. With respect to differential integrated circuits, in [9], for example, the common-mode gain of the classic $\mu \mathrm{A} 741$ op amp was assumed to have a 
single pole (at the same frequency as the differential gain) and a single zero and the measured CMRR modulus actually showed a "low-pass" variation with frequency (due to the zero of the common-mode gain). No information about the phase characteristic was provided. Similarly, a detailed study of CMOS operational transconductance amplifiers showed that their common-mode gain had the same dominant pole of the differential gain and two zeros, so that "the CMRR frequency response is basically determined by two poles which correspond to the two zeros of the common-mode gain." [10]. The agreement between simulations and calculations in [10] were excellent. The amplifier in [5] shows a common-mode gain with a pole and a zero, and a CMRR that decreases about $20 \mathrm{~dB} /$ decade. Therefore, if the CMRR for differential circuits, integrated or not, decreases inside the $-3 \mathrm{~dB}$ bandwidth of the differential gain, this means that the commonmode gain increases inside that frequency band, yet this gain cannot be deducted from the modulus of the CMRR, even if the frequency dependence of the differential gain is known in detail, so that its subtleties remain hidden to the user. As a result, CMRR calculations for cascaded differential stages that assume that this parameter is a real number within the frequency band of the differential signal being amplified [11] [12] cannot be directly applied inside all the differential amplifier passband.

Furthermore, for fully-differential circuits built from separated stages like that in Fig. 1, the differential to common-mode gain is $G_{\mathrm{CD}}(\mathrm{s})=G_{\mathrm{DC}}(\mathrm{s}) / 4$ [4]. Therefore, the effects of the input differential signal on the output common mode signal are qualitatively the same described for $G_{\mathrm{DC}}$ in Section II. This implies that the output common-mode signal comprises the input common mode signal amplified by $G_{\mathrm{CC}}(\mathrm{s})\left(=G_{\mathrm{DD}}(\mathrm{s})\right)[4]$, plus the input differential signal converted into common-mode output through $G_{\mathrm{CD}}(\mathrm{s})$, with its band-pass or low-pass response. Consequently, the ensuing stage will need to tackle a larger common mode signal than the input stage, amplified 
because of $G_{\mathrm{CC}}(\mathrm{s})$, and distorted, because of $G_{\mathrm{CD}}(\mathrm{s})$.

\section{CONCLUSIONS}

The deleterious effects of common-mode signals in differential measurements cannot be predicted from the CMRR modulus because this parameter is not a transfer function but the quotient of two transfer functions, namely the differential-mode and the common-mode gains. Consequently, that prediction needs the gain and phase characteristic of the common-mode gain, hence these should be specified or measured. Otherwise, a flat CMRR (in decibels) in a given frequency band, for example, could be wrongly interpreted as a real number when in fact it is a complex quantity with a phase that cannot be predicted from the modulus and that deviates from $0^{\circ}$ well below the corner frequency of the differential mode gain.

Since the common-mode gain arises from component mismatching due to fabrication tolerances, and these are worse for passive discrete components, high-performance differential signal conditioners should not involve separate signal chains for each of the two signals to be processed and eventually subtracted, like the circuit in Fig. 1. Component tuning can help in improving the dc performance but at the cost of worsening ac performance. Multi-channel systems, in particular, wherein component tuning is unfeasible, should avoid circuit topologies that make the commonmode response to depend on passive component matching. Since the common-mode gain increase, when present, starts inside the circuit passband, designing a corner frequency much higher than the signal bandwidth could alleviate the problem but at the cost of increased noise bandwidth.

Common-mode gain in IC differential components such as op amps and instrumentation amplifiers may not obey the equations obtained for the circuit in Fig. 2, where mismatched passive components determine the common mode gain. Nevertheless, the qualitative conclusions about the results of subtracting two dissimilar first-order transfer functions apply whatever the circuit 
topology. Therefore, by measuring the common-mode gain, Table I can be used to identify the source and relevance of the mismatch.

\section{ACKNOWLEDGMENT}

The authors wish to thank Prof. Ørjan Martinsen (University of Oslo) for pointing out that common-mode gain could be a major error source in low-frequency bioimpedance measurements. The authors also thank the Castelldefels School of Telecommunications and Aerospace Engineering (EETAC-UPC) for its research facilities, and Mr. F. López for his technical support.

\section{REFERENCES}

[1] Pallàs Areny R, Webster J G. Sensors and Signal Conditioning, $2^{\text {nd }}$ ed. New York: John Wiley \& Sons, 2001.

[2] Nasserian M, Peiravo A, Moradi A. A fully-integrated 16-channel EEG readout front-end for neural recording applications. AEU - International Journal of Electronics and Communications, 2018; 94: 109-121. https://doi.org/10.1016/j.aeue.2018.06.045

[3] Wanga J J, Chena D Y, Wang S F, Wei R S, A multi-band low noise amplifier with wide-band interference rejection improvement. AEU - International Journal of Electronics and Communications, 2016; 70: 320-325. https://doi.org/10.1016/j.aeue.2015.12.010

[4] Pallàs Areny R, Webster J G. Common-mode rejection ratio in differential amplifiers. IEEE Trans. Instrum. Meas., 1991: 40:669-676. https://doi.org/10.1109/19.85332

[5] Ben-Esmael M, Hart B.L, Hayatleh K, Lidgey F.J. CMRR-bandwidth extension technique for CMOS differential amplifiers. AEU - International Journal of Electronics and Communications, 2014; 68, 990-993. https://doi.org/10.1016/j.aeue.2014.04.022 
[6] White D R. Phase compensation of the three-op-amp instrumentation amplifier. IEEE Trans. Instrum. Meas., 1987; 36,: 842-844. https://doi.org/ 10.1109/TIM.1987.6312800

[7] Gasulla M, Casas O, Pallàs-Areny R. On the common mode response of fully differential circuits, Proc. $17^{\text {th }}$ IEEE IMTC, Baltimore, 2000, pp. 1045-1049. . https://doi.org/10.1109/IMTC.2000.848900

[8] Casas O, Pallàs Areny R. Basics of analog differential filters. IEEE Trans. Instrum. Meas., 1996; 45: 275-279. https://doi.org/10.1109/19.481347

[9] Brinson M E, Faulkner D J. New approaches to measurement of operational amplifier common-mode rejection ratio in the frequency domain. IEE Proc. Circuits Devices Syst., 1995 142:247-253. https://doi.org/10.1049/ip-cds:19952031

[10] Giustolisi G, Palmisano G, Palumbo G. CMRR frequency response of CMOS operational transconductance amplifiers. IEEE Trans. Instrum. Meas, 2000; 49: 137-143. . https://doi.org/10.1109/19.836324

[11] Pallàs Areny R, Webster J. G. Common-mode rejection ratio for cascaded differential amplifier stages. IEEE Trans. Instrum. Meas., 1991; 40:.677-680. . https://doi.org/10.1109/19.85333

[12]Koide F T. Common-Mode Rejection Ratio redefined. IEEE Trans. Instrum. Meas.,1997; 46:737-739. https://doi.org/ 10.1109/19.585444

[13]Steyaert M.S.J., Sansen W.M.C., A micropower low-noise monolithic instrumentation amplifier for medical purposes. IEEE Journal of Solid State Circuits, 1987, 22: 1163-1168,. https://doi.org/10.1109/JSSC.1987.1052869 


\section{Figures and tables}

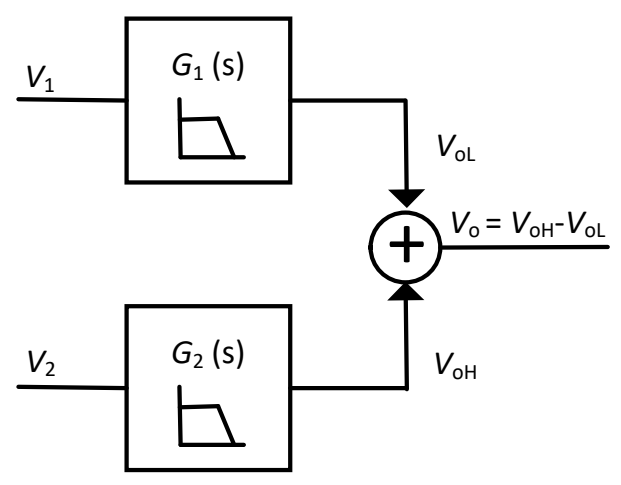

Fig. 1. Differential circuit built by subtracting the output voltages of two single-ended circuits.

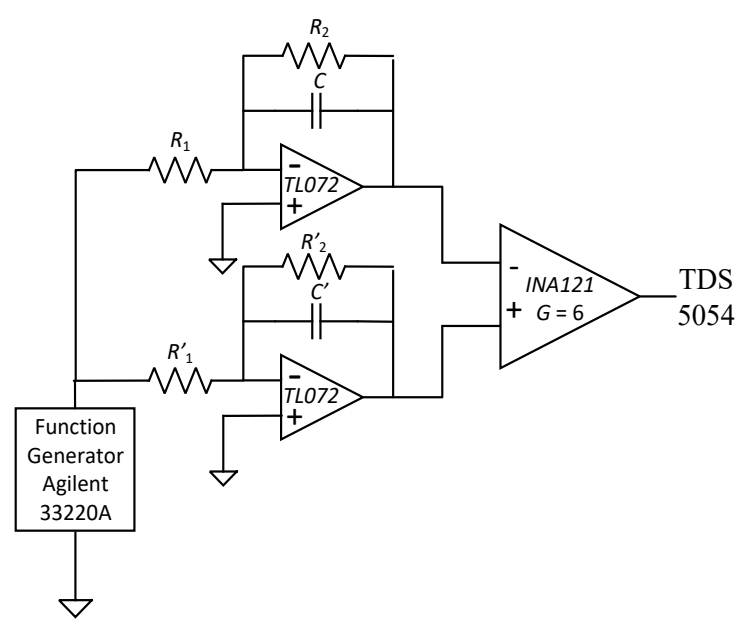

Fig. 2. Differential amplifier built by subtracting the output voltages of two single-ended amplifiers that are mismatched because of component tolerance.
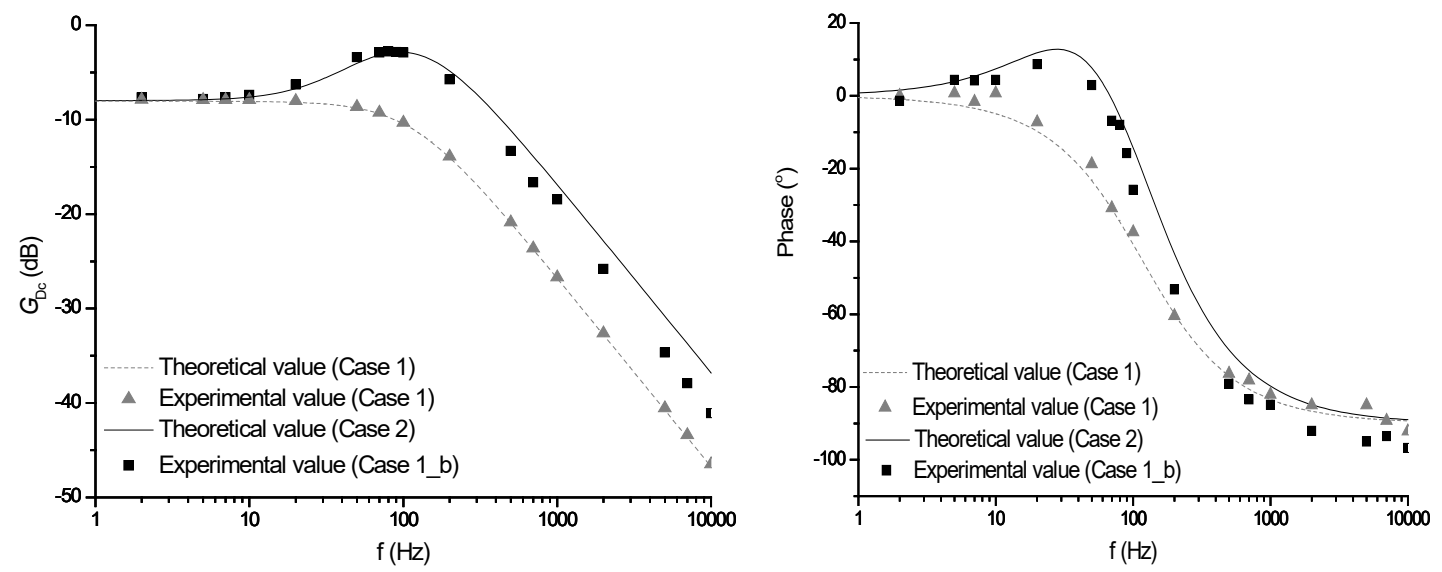

Fig. 3. $G_{\mathrm{DC}}$ modulus (left) and phase (right) for Cases 1 and 2 in Table III. Case 1: $G_{2}>G_{1}$ and $t_{\omega}<<t_{G}$; Case 2: $G_{2}>$ $G_{1}$ and $t_{\omega}>t_{G}$. 

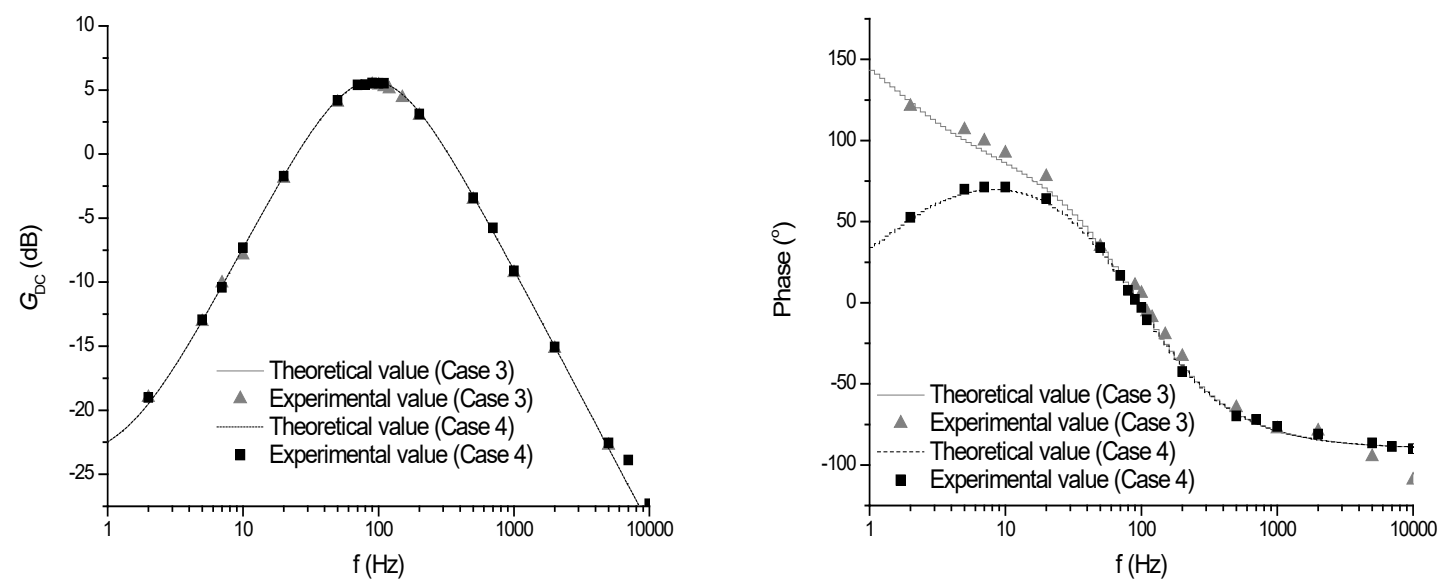

Fig. 4. $G_{\mathrm{DC}}$ modulus (left) and phase (right) for Cases 3 and 4 in Table III. Case 3: $G_{2}<G_{1}$ and $\omega_{2}>\omega_{1}$; Case 4: $G_{2}<$ $G_{1}$ and $\omega_{2}<\omega_{1}$. In both cases, $t_{\omega}>>t_{G}$.

TABLE I

COMMON-MODE GAIN TRANSFER FUNCTIONS DEPENDING ON MISMATCHES

\begin{tabular}{|c|c|c|}
\hline $\begin{array}{l}G_{2}>G_{1} \\
\omega_{2}>\omega_{1} \\
\omega_{2}<\omega_{1}{ }^{*}\end{array}$ & $t_{G} \neq 0$ & $t_{G}=0$ \\
\hline$t_{\omega} \neq 0$ & & Band-pass response (17) \\
\hline$t_{\omega}>0.4 t_{G}$ & $\begin{array}{c}\text { Band-pass response }(9),{ }^{*}(20) \\
\text { Gain peaking } \\
\left|G_{\mathrm{DC}}\right|_{\max } \approx G_{\mathrm{a}}\left(t_{G}+t_{\omega}\right) \\
\phi>0 \text { from } \omega_{\mathrm{zc}} \text { up to past } \omega_{1}\end{array}$ & \\
\hline$t_{\omega}<0.4 t_{G}$ & $\begin{array}{c}\text { Low-pass response (9) } \\
\qquad \begin{array}{c}\left|G_{\mathrm{DC}}(\omega)\right| \leq \Delta G \\
\phi<0\end{array}\end{array}$ & $\begin{array}{c}\text { Gain peaking } \\
\left|G_{\mathrm{DC}}\right|_{\max }=G_{\mathrm{a}} t_{\omega} \\
\phi>0 \text { up to past } \omega_{1}\end{array}$ \\
\hline$t_{\omega}=0$ & $\begin{array}{c}\text { Low pass response (18) } \\
\qquad \phi<0\end{array}$ & $G_{\mathrm{DC}}=0$ \\
\hline$G_{1} \omega_{1}=G_{2} \omega_{2}$ & \multicolumn{2}{|c|}{$\begin{array}{c}\text { Second-order, low-pass response (22) } \\
\phi<0\end{array}$} \\
\hline
\end{tabular}

TABLE II

COMPONENTS FOR THE CIRCUIT IN FIG. 2 IMPLEMENTATIONS

\begin{tabular}{lcccc}
\hline & Case 1 & Case 2 & Case 3 & Case 4 \\
\hline$R_{1} / \mathrm{k} \Omega$ & 10.35 & 10.35 & 9.91 & 9.91 \\
$R_{2} / \mathrm{k} \Omega$ & 99.71 & 99.71 & 99.30 & 99.30 \\
$R_{1} / \mathrm{k} \Omega$ & 9.91 & 9.91 & 9.87 & 9.87 \\
$R_{2} / \mathrm{k} \Omega$ & 99.35 & 99.35 & 99.60 & 99.60 \\
$C / \mathrm{nF}$ & 14.57 & 16.06 & 14.59 & 21.30 \\
$C ' / \mathrm{nF}$ & 14.59 & 14.59 & 21.30 & 14.59 \\
& & & & \\
\hline \hline
\end{tabular}


TABLE III

Circuit PARAMETERs For THE COMPONENT VALUeS IN TABLE II

\begin{tabular}{lrrrr}
\hline \hline & Case 1 & Case 2 & Case 3 & Case 4 \\
\hline$G_{1}$ & 9.63 & 9.63 & 10.03 & 10.03 \\
$G_{2}$ & 10.03 & 10.03 & 10.09 & 10.09 \\
$G_{\mathrm{a}}$ & 9.83 & 9.83 & 10.06 & 10.06 \\
$\Delta G$ & 0.40 & 0.40 & 0.06 & 0.06 \\
$t_{G} / \%$ & 2 & 2 & 0.3 & 0.3 \\
$f_{1} / \mathrm{Hz}$ & 109.53 & 99.39 & 75.02 & 109.45 \\
$f_{2} / \mathrm{Hz}$ & 109.78 & 109.78 & 109.78 & 75.25 \\
$f_{\mathrm{a}} / \mathrm{Hz}$ & 109.65 & 104.59 & 92.40 & 92.35 \\
$\Delta f / \mathrm{Hz}$ & 0.25 & 10.39 & 34.76 & 34.20 \\
$\Delta \omega / \mathrm{rad} / \mathrm{s}$ & 1.57 & 65.28 & 218.38 & 214.88 \\
$t_{\omega} / \%$ & 0.11 & 5 & 18.8 & 18.5 \\
$t_{\omega} / t_{G}$ & 0.055 & 2.50 & 62.70 & 61.70 \\
$f_{\mathrm{zc}} / \mathrm{Hz}$ & 103.69 & 29.83 & 1.50 & 1.87
\end{tabular}

TABLE IV

ESTIMATED $G_{\mathrm{DC}}$ AND CMRR FOR DIFFERENT GAINS AND TOLERANCES

\begin{tabular}{lrrrr}
\hline \hline & Case A & Case B & Case C & Case D \\
\hline$G_{\mathrm{a}}$ & 10 & 100 & 100 & 10 \\
$t_{G} / \%$ & 0.01 & 0.01 & 0.01 & 0.01 \\
$f_{\mathrm{a}} / \mathrm{kHz}$ & 1 & 1 & 1 & 1 \\
$t_{\omega} / \%$ & 10 & 10 & 1 & 0.1 \\
$t_{\omega} / t_{G}$ & 1000 & 1000 & 100 & 10 \\
$f_{\mathrm{zc}} / \mathrm{Hz}$ & 0.99 & 0.99 & 9.90 & 90.91 \\
$G_{\mathrm{DC}}(0) / \mathrm{dB}$ & -54 & -34 & -34 & -54 \\
$G_{\mathrm{DC}(\max )} / \mathrm{dB}$ & 0 & 20 & 0 & -39 \\
$\phi_{\mathrm{DC}(\max )} /{ }^{\circ}$ & 85 & 85 & 74 & 43 \\
$f\left(\phi_{\mathrm{DC}(\max )}\right) / \mathrm{Hz}$ & 21 & 21 & 76 & 191 \\
$\mathrm{CMRR}(0) / \mathrm{dB}$ & 74 & 74 & 74 & 74 \\
$\mathrm{CMRR}$ & 17 & 17 & 37 & 56 \\
$\phi_{\mathrm{CMRR}(\max )} /{ }^{\circ}$ & -86 & -86 & -79 & -56 \\
$f\left(\phi_{\mathrm{CMRR}(\max )}\right) / \mathrm{Hz}$ & 30 & 30 & 96 & 305
\end{tabular}

